\title{
The cardiovascular effects of midazolam co-induction to propofol for induction in aged patients
}

\author{
Young Soo Lim, Dong Hee Kang, Se Hwan Kim, Tae Ho Jang, Kyung Han Kim, Sie Jeong Ryu, \\ Soo Bong Yu, and Doo Sik Kim
}

Department of Anesthesiology and Pain Medicine, Kosin University College of Medicine, Busan, Korea

Background: The aim of this study was to investigate whether a small dose of midazolam and lessening the propofol dosage could prevent cardiovascular change at tracheal intubation for induction in aged patients.

Methods: Eighty patients over 65 years (ASA physical status 1,2) scheduled for elective surgery received general anesthesia with remifentanil and propofol or midazolam. Patients in group $\mathrm{P}(\mathrm{n}=40)$ were induced with $0.9 \% \mathrm{NaCl}$ $0.03 \mathrm{ml} / \mathrm{kg}$, propofol $1.2 \mathrm{mg} / \mathrm{kg}$ and remifentanil. Patients in group MP ( $\mathrm{n}=40)$ were induced with midazolam 0.03 $\mathrm{mg} / \mathrm{kg}$, propofol $0.8 \mathrm{mg} / \mathrm{kg}$ and remifentanil. The time taken to reach loss of consciousness (LOC) and the value of bispectral index score (BIS) at LOC were recorded. After LOC, $0.8 \mathrm{mg} / \mathrm{kg}$ of rocuronium was given and tracheal intubation was performed. The mean blood pressure (MBP) and heart rate (HR) were recorded before induction as the base value, before intubation, immediately post-intubation and 3 minutes after intubation.

Results: Compared with the base values, MBP at before intubation and 3 minutes after intubation was significantly decreased in group $\mathrm{P}$ and group MP $(\mathrm{P}<0.05)$. Compared with group $\mathrm{P}$, the decrease of MBP was significantly less at before intubation, immediately after intubation and 3 minutes after intubation in group $\mathrm{MP}(\mathrm{P}<0.05)$. The time taken to reach LOC was significantly decreased in group MP compared with that in group $\mathrm{P}(\mathrm{P}<0.05)$. There were no significant differences of HR at any time between the two groups.

Conclusions: Co-induction with midazolam and propofol could prevent a marked BP decrease at tracheal intubation for induction in aged patients. (Korean J Anesthesiol 2012; 62: 536-542)

Key Words: Aged, Cardiovascular system, Drug synergism, Midazolam, Propofol.

\footnotetext{
Received: June 15, 2011. Revised: 1st, July 21, 2011; 2nd, September 23, 2011; 3rd, October 25, 2011; 4th, November 2, 2011. Accepted: November 2, 2011. Corresponding author: Doo Sik Kim, M.D., Department of Anesthesiology and Pain Medicine, Kosin University College of Medicine, 34, Amnam-dong, Seo-gu, Busan 602-702, Korea. Tel: 82-51-990-6283, Fax: 82-51-254-2504, E-mail: kds0728md@gmail.com

(c) This is an open-access article distributed under the terms of the Creative Commons Attribution Non-Commercial License (http:// creativecommons.org/licenses/by-nc/3.0/), which permits unrestricted non-commercial use, distribution, and reproduction in any medium, provided the original work is properly cited.
} 


\section{Introduction}

Aged patients require very careful attention and observation for induction of anesthesia and endotracheal intubation because they are likely to have high frequency of side effects even with an adult dose due to the deterioration of organ function, change in autonomic nervous system and increase of drug sensitivity due to aging in comparison to young people [1]. In addition, in aged patients, special attention is required to avoid any serious decrease of heart rate (HR) because the risks of cardiac ischemia, cerebral ischemia and cerebral infarction may increase even from temporary decreases of blood pressure (BP) and HR. Propofol being used for induction of general anesthesia has a rapid of onset time; it is therefore recommended intravenously infused anesthetic agent for anesthesia of the aged. However in aged patients, the sensitivity of propofol for the brain needs to be increased therefore the anesthetic effects are manifested as increased at the same dose. [2]. Also a problem arises that when inducing the anesthesia using propofol, whereby the prominent decrease of systolic pressure by $15-40 \%$ may be more prevalent in the aged [3].

Therefore, in the aged patients whose hemodynamic changes are severe, the use of the co-induction method with an intravenously infused anesthetic agent can escalate the efficacy and the safety of the drug agent $[4,5]$. Co-induction refers to a method in which the anesthetic agent is administered together with a small quantity of sedatives or another anesthetic agent in order to reduce the dosage of the anesthesia induction agent. This method is advantageous as it minimize the side effects that can be manifested for induction of anesthesia with the same or higher efficacy by using two or more drug agents as a combination compared to using single agent [4].

Propofol and remifentanil are the drug agents recently being used for co-induction [6]. When inducing the general anesthesia by co-administration of propofol and remifentanil, the optimal dose of each agent should be well selected; otherwise, side effects such as hypotension and bradycardia can result $[6,7]$. Especially, incidence of a severe BP decrease before and after endotracheal intubation is frequently encountered in aged patients evev when the minimum dose required for induction of anesthesia had been used.

Although it has been known that the co-administration of midazolam and propofol can reduce the inductive dosage of propofol by synergism of the narcotic effect $[5,8]$, there has been no report in actual conditions on cardiovascular changes incurring from the co-administration of midazolam and propofol for induction of anesthesia in aged patient in Korea. Therefore, this study aimed to identify whether the induction of general anesthesia performed by the co-administration of propofol and remifentanil in aged patients can reduce the dosage of propofol and whether co-administration with small amount of midazolam can not only reduce the inductive dosage of propofol but also reduce the cardiovascular changes being manifested before and after the endotracheal intubation.

\section{Materials and Methods}

This study was initiated after obtaining Hospital Ethics Committee approval and written informed consent from all subject patients on the day before the surgery after explaining to them the objectives and methods of this study. This study was performed in 80 American Society of Anesthesiologists (ASA) physical status class I-II patients whose ages were over 65 years and who were scheduled for elective surgery under general anesthesia. Any patient with a history of hypertension or diabetes mellitus, or currently under treatment for respiratory diseases, patients with a history of myocardial infarction or cerebral infarction, patients with dementia or psychiatric patients, patients under dialysis treatment due to renal failure or patients of whom it is difficult to perform the endotracheal intubation at over Mallampati class 3 are all excluded from participation in this study.

The subject patients fasted for over 8 hours before the surgery and no premedication was given. After a patient was admitted to the operation room, an electrocardiogram (ECG), non-invasive manometer (Philips MP40), and apulse oximeter and bispectral index (BIS) sensor (BISTM quatro, Aspect Medical System, USA) were attached to the patient.

After the patient was stabilized, the mean BP and HR as well as pulse saturation were measured as a baseline. As premedication, $0.2 \mathrm{mg}$ of glycopyrrolate was infused by intravenous administration and for preoxygenation before induction of anesthesia all patients were asked to deeply breathe $10 \mathrm{~L} / \mathrm{min}$ oxygen 8 times for 1 minute [9]. Although deep breathings of $100 \%$ oxygen 4 times, for 30 seconds is the proper procedure for young and healthy patients, in the aged patient in which desaturation is rapid, the time for desaturation needed to be extended in order to by filling not only the pulmonary alveolus and arteries but also the tissues and veins. In this study, in order to maximize oxygenation in the shortest amount of time, when applying the aforementioned procedure, during the operation, the oxygen saturation was maintained at over $99 \%$ in all patients.

Anesthesia was initiated with $10 \mu \mathrm{g} / \mathrm{kg} / \mathrm{hr}$ remifentanil using a drug infusion pump. The subjects were randomly assigned to two groups; one group with the co-administration of midazolam and propofol and the other group with the administration of propofol alone as the single agent. The groups were set as the test group (MP group, $\mathrm{n}=40$ ) and the control group (P group, $\mathrm{n}=40$ ). In the group MP test group, midazolam $0.03 \mathrm{mg} / \mathrm{kg}$ 
was intravenously infused simultaneously with the initiation of remifentanil administration and propofol was added as $0.8 \mathrm{mg} /$ $\mathrm{kg}$ after 2 minutes, whereas in the group-P control group, $0.9 \%$ $\mathrm{NaCl} 0.03 \mathrm{ml} / \mathrm{kg}$ was intravenously infused simultaneously with the initiation of remifentanil administration in addition to the administration of propofol at $1.2 \mathrm{mg} / \mathrm{kg} 2$ minutes later.

The unconsciousness was confirmed by the non-reaction of patients to the verbal order "Please open your eyes" together with the loss of lid reflex. Also the BIS value of the time was recorded.

A muscle relaxant, rocuronium $0.8 \mathrm{mg} / \mathrm{kg}$, was intravenously injected 60 seconds after the administration of propofol and bag-valve ventilation was performed for 1 minute and 30 seconds. Rocuronium used as a muscle relaxant for the induction of anesthesia was required at the dosage of $0.6 \mathrm{mg} /$ $\mathrm{kg}$ for endotracheal intubation; nonetheless, it was used with an increased dose of $0.8 \mathrm{mg} / \mathrm{kg}$ to ensure sufficient muscle relaxation in consideration of clinical conditions in which it is difficult to measure TOF for assessing the muscle relaxation status.

After confirmation of sufficient muscle relaxation, the endotracheal intubation was carried out by a resident with over 3 years experience. 5 minutes after the initial administration of remifentanil after endotracheal intubation was performed, remifentanil was administered with a reduced dose at $2 \mu \mathrm{g} / \mathrm{kg} /$ $\mathrm{hr}$ and the anesthesia was maintained with sevoflurane $1.5 \%$, oxygen at $1.5 \mathrm{~L} / \mathrm{min}$ and air at $2.5 \mathrm{~L} / \mathrm{min}$. Also mechanical ventilation was carried out in order to maintain $\mathrm{PETCO}_{2}$ at $30-$ $35 \mathrm{mmHg}$.

Mean blood pressure (MBP) and HR were measured and recorded at the baseline before induction of anesthesia (B) immediately before performing endotracheal intubation (BI), immediately after performing endotracheal intubation (PI0) and at 3 minutes after performing endotracheal intubation (PI3). It was decided that when the patient's HR had decreased below 45 times per minute, atropine $0.5 \mathrm{mg}$ was to be intravenously injected, whereas when the systolic blood pressure of the

Table 1. Patient Characteristics

\begin{tabular}{lcc}
\hline & $\begin{array}{c}\text { Group MP } \\
(\mathrm{n}=40)\end{array}$ & $\begin{array}{c}\text { Group P } \\
(\mathrm{n}=40)\end{array}$ \\
\hline Gender (M/F) & $19 / 21$ & $23 / 17$ \\
Age (yr) & $70.3 \pm 4.2$ & $68.3 \pm 3.3$ \\
Height $(\mathrm{cm})$ & $163.5 \pm 9.4$ & $162.4 \pm 7.8$ \\
Weight $(\mathrm{kg})$ & $62.6 \pm 9.1$ & $60.8 \pm 8.9$ \\
BMI $\left(\mathrm{kg} / \mathrm{m}^{2}\right)$ & $23.4 \pm 2.3$ & $23.0 \pm 2.4$ \\
\hline
\end{tabular}

Values are mean \pm SD or number of patients. Group MP: coinduction with midazolam $0.03 \mathrm{mg} / \mathrm{kg}$ and propofol $0.8 \mathrm{mg} / \mathrm{kg}$, Group P: induction with propofol $1.2 \mathrm{mg} / \mathrm{kg}$ only. BMI: body mass index. patient after performing endotracheal intubation was lower $80 \mathrm{mmHg}$, ephedrine was to be given through intravenous administration with an increase of dose by $5 \mathrm{mg}$.

For statistical analysis, the study used SPSS (version 13.0, SPSS Inc, Chicago, USA) and all results were indicated as mean \pm standard deviation. Inter-group comparison by time points was conducted using an unpaired T-test and the intra-group variations of MBP and HR according to time were analyzed by using repeated ANOVA measures. If the $\mathrm{P}$ value was less than 0.05 , it was determined as statistically significant.

\section{Results}

There was no inter-group significant difference in age, gender, height, body weight, BMI and in the ASA physical status grading classification (Table 1). Also, there was no inter-group significant difference observed of $\mathrm{BP}$ and $\mathrm{HR}$ at the baseline. For the time taken until the loss of consciousness after intravenous administration of propofol, the MP group (38 \pm 10.8$)$ showed a significantly shorter time compared to the P group (53 \pm 12.1 , but there was no inter-group significant difference in BIS value at the time of loss of consciousness.

For the mean BP, the MP group showed significant small decreases of BP at BI, PI0 and PI3, compared to the P group. The MP showed significant decreases of BP at BI (21.0\%) and PI3 (15.3\%), compared to those at the baseline, and the P group also showed prominent decreases of BP at BI (29.7\%) and PI3 (23.2\%), compared to those at the baseline (Fig. 1).

The HR showed lower values overall in the MP group than in the $\mathrm{P}$ group from the inter-group comparison but had no

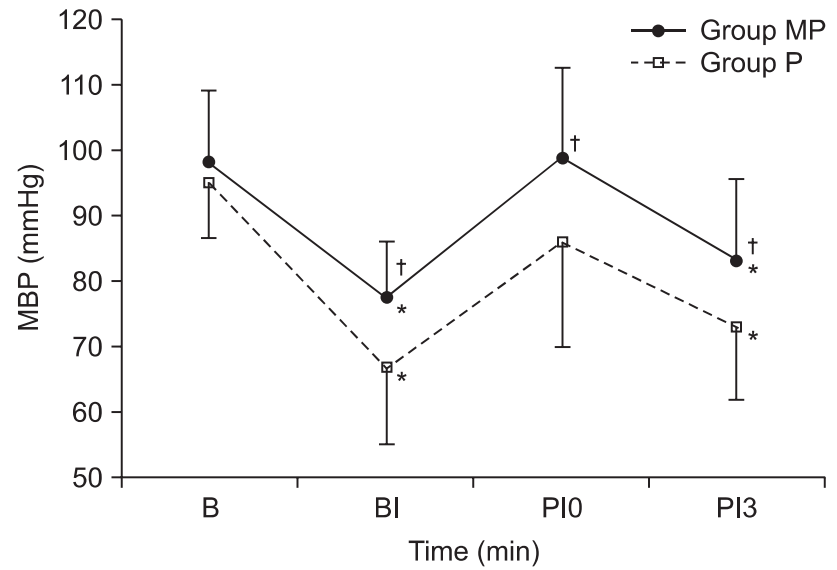

Fig. 1. Changes of mean blood pressure (MBP) during the induction of anesthesia. Values are mean \pm SD. Group MP: midazolam + propofol, Group P: only propofol. B: baseline, BI: before intubation, PI0: just post intubation, PI3: 3 minutes after intubation. $* \mathrm{P}<0.05$, compared with baseline within each group, ${ }^{\dagger} \mathrm{P}<0.05$, compared with Group P. 


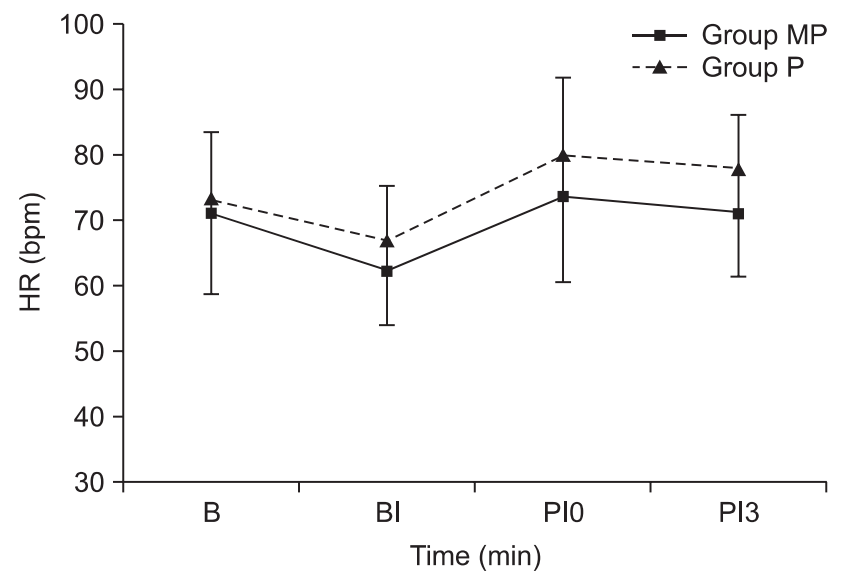

Fig. 2. Changes of heart rate (HR) during the induction of anesthesia. Values are mean \pm SD. Group MP: midazolam + propofol, Group P: only propofol. B: baseline, BI: before intubation, PI0: just post intubation, PI3: 3 minutes after intubation.

statistically significant difference. Also, from both the MP group and $\mathrm{P}$ groups, there was no significant difference in the HR by each time point (Fig. 2).

Although bradycardia requiring the use of atropine had not incurred in both groups, 3 patients of the group administered with $1.2 \mathrm{mg} / \mathrm{kg}$ propofol had shown a decrease of systolic BP; they were thereby excluded from the study subjects after intravenous injection of ephedrine $5 \mathrm{mg}$.

\section{Discussion}

In the results of this study, it was identified that the group with the co-induction of general anesthesia with a combination of midazolam and propofol in aged patients did not have large scaled decreases of MBP at the time point of immediately before performing endotracheal intubation as well as at the time points of 1 minute and 3 minutes after performing endotracheal intubation, in comparison to the group with a single induction of propofol only $(\mathrm{P}<0.05)$. Even compared to the BP at the baseline, the group with co-induction did not show any increase of mean BP at the time point of 1 minute after performing endotracheal intubation. To this end, it is considered that using midazolam as a co-induction drug in combination with propofol for the induction of anesthesia in aged patients, can prevent sudden BP decreases incurring immediately before, and at the time point of 3 minutes after, performing endotracheal intubation as well as the notable BP decreases incurring between immediately after the endotracheal intubation and at 3 minutes after endotracheal intubation with midazolam.

During the operation, the sympathetic tone increases in the patients, causing cardiovascular change [10], and in particular, the variation is presented in larger scale in aged patients. Paterniti et al [11], in their study on the physiologic characteristic of aged patients, found that physical organic dysfunction and incurrence of additional diseases had increased in proportion to age, and that in their central nervous system functions, cardiovascular functions, hepatic functions, renal functions and pulmonary functions were reduced, resulting in a high ratio of pre and postoperative morbidity as well as mortality. Ryu [1] also suggested that in aged patients, the organic functions were decreased while cardiovascular functions were increased in addition to new incurrences of diseases affecting pharmacodynamics and pharmacokinetics including diabetes mellitus and abnormality in renal function along with the aging symptoms; therefore, even if the same dose of drug agent was administered, the drug effects would be presented as increased due to the increase of drug-sensitivity, in addition to the highly likely frequency of side effects. Moreover, the non-compliant heart of aged patients also led to a large scale variation in ventricular preload and cardiac output as responses to even small variations of venous return. Therefore, Ryu had suggested that aged patients do not have a good level of compensation to the decrease of blood volume due to notable reductions in diastolic myocardial function, the control of the HR by a baroreceptor, adrenergic receptor responsiveness and vascular compliance [1]. Propofol, as alkylphenol group drug agent, presents direct myocardial depression effects through the negative inotropic effect [8] and cardiovascular inhibition effects through the reduction of systemic vascular resistance by vasodilative action [12]. The administration of a suitable dose for the induction of anesthesia inhibits the baroreflex mechanism, inhibiting the acceleration of HR due to the decrease of arterial pressure. These results may be worsened even further by factors such as a large dosage, infusion at a rapid speed, and old ages.

Moreover, in aged patients, the blood concentration of propofol could be maintained at a high level as the patients had quite a low clearance rate of propofol and small central compartment capacity [13]; the conditions allow the cardiovascular inhibition effect to be expressed more prominently in aged patients. Therefore, when performing the induction of anesthesia using propofol on aged patients or on patients whose cardiovascular system is unstable, a careful readiness for the procedure is required with a preemptive expectation of a high prevalence of serious BP decreases in proportion with IV doses and speeds among aged patients in comparison to young patients $[3,14]$. In this study, it took 5 minutes from the administration of propofol until the BP measurement after the endotracheal intubation and it was considered that BP had not reached the lowest BP incurring at the post- propofol administration due to stimulation by the endotracheal intubation [1]. It is expected that a serious BP decrease might have incurred if measured at 5 minutes after the endotracheal 
intubation under the conditions of no stimulation. However, it was determined that a serious BP decrease or a prolonged time of such decrease was likely to have harmful effects on the aged patient, and that there was a realistic limitation in idly waiting for 5 minutes under the conditions until processing the invasive procedure or surgery after the endotracheal intubation. Moreover, because the results presented a significant intergroup difference in $\mathrm{BP}$ variation trends for 3 minutes after the endotracheal intubation, BP was measured only up to 3 minutes after the endotracheal intubation. Charlson et al. [15] had reported that the decrease of intra-operative BP (decrease by more than $30 \%$ from the arterial pressure before induction of anesthesia) had caused reinfrarction in $20 \%$ of patients who had myocardial infraction; in addition the postoperative complication of ischemic heart disease was shown with a high prevalence when the mean myocardial decrease of more than $20 \mathrm{mmHg}$ had been sustained for 5 to 59 minutes. Therefore, when performing the induction of anesthesia in aged patients, measures should be taken to prepare for the possible incidence of a serious BP decrease within 5 minutes after the endotracheal intubation, and if such BP decrease is incurred, the time for sustenance of low blood pressure status should be shortened by using a position change or inotropics.

From a preliminary study conducted prior to this study in aged patients over 65 years old, a few patients did not reach unconsciousness when administered with propofol $1.0 \mathrm{mg} / \mathrm{kg}$, and a few patients showed a decrease of systolic pressure below $70 \mathrm{mmHg}$ when administered $1.4 \mathrm{mg} / \mathrm{kg}$ propofol. Given these results, in this study, the administration dose of propofol for the group with propofol only was set as $1.2 \mathrm{mg} / \mathrm{kg}$. Schüttler and Ihmsen [16] recommended using propofol by reducing its dose for adults by over $40 \%$ because the clearance rate of propofol could be reduced in aged patients over 60 years old. On the other hand, Olmos et al. [17] reported that the required dose of propofol was reduced by $37 \%$ in patients in the over 60 years old group who had not received premedication for induction of anesthesia by controlled injection of target concentration in comparison to the under 40 years old patients. They also reported that as the age increased by 10 years, the required dose was to be reduced accordingly. In addition, as aged patients are sensitive due to the reduction of total body clearance rate according to the pharmacokinetic variation, it is important to increase or decrease the dose of medication depending on the age. Generally, it is recommended that a medication which can maintain the cardiopulmonary functions safely when the patient is over 40 years old should be administered by reducing the medication dose by 10 to $12 \%$ at each 10 year increase and that medication should be used that presents a short action time and fast recovery [18-21]. In this study, the dose of $1.2 \mathrm{mg} /$ $\mathrm{kg}$ propofol for the group with propofol only was administered by the reduction of the adult dose by $20-52 \%$, which was considered as $1.5-2.5 \mathrm{mg} / \mathrm{kg}$.

Midazolam is a water-soluble benzodiazepine with a fast onset of action of 2 to 3 minutes and a short distribution half-life of 5 to 10 minutes. It has been widely used recently as the premedication agent of anesthesia because it was effective in resolving anxiety of surgery and in stabilizing the cardiovascular system during the perioperative period $[22,23]$. When performing the co-induction of anesthesia by combining midazolam with propofol, the single intravenous bolus administration of propofol or its gradual additional intravenous administration had shown the synergistic action of sleep effectiveness, indicating that such co-induction presents the effect of approximately $25-35 \%$ higher dose reduction in comparison to single respective doses of each medication [24]. Kim et al. [8] reported that the prior administration of $2 \mathrm{mg}$ $(0.032 \mathrm{mg} / \mathrm{kg})$ midazolam combined with sustained intravenous infusion of propofol reduced the sleep-inducing dose of propofol by $29 \%$. To this end, in this study, instead of setting the administration dose of midazolam as $0.03 \mathrm{mg} / \mathrm{kg}$ in the MP group, the administration dose of propofol had been reduced to $0.8 \mathrm{mg} / \mathrm{kg}$. Unlike this study, where the single intravenous bolus administration has been used, Teh et al. [25] reported that the cause of the sleep-effect synergistic action of midazolam and propofol (in the studies in which the continuous IV infusion method was used), was the pharmacodynamics interaction between the two drug agents at the $\mathrm{GABA}_{\mathrm{A}}$ of brain but with no synergistic action of the cardiovascular system. The objective of this study was to identify not only the synergistic action on the sleep effect but also the hemodynamic synergistic action against the inhibition of the cardiovascular system. The study result showd that the co-induction of anesthesia in aged patients by a combination of midazolam and propofol, had synergistic actions in terms of sleep-effect as well as hemodynamic effects. BIS was used to assess the unconsciousness. The BIS values at the time of falling into unconsciousness had no significant inter-group difference between the two groups, but the time taken to reach unconsciousness was significantly more rapid in the MP group than in the P group. This demonstrates that the co-induction of anesthesia by the combination of midazolam and propofol had shown synergistic action.

In this study, remifentanil was administered at a certain rate of $10 \mu \mathrm{g} / \mathrm{kg} / \mathrm{hr}$ from the beginning of the anesthesia induction until the performing of endotracheal intubation and it was administered at a reduced amount of $2 \mu \mathrm{g} / \mathrm{kg} / \mathrm{hr}$ after 5 minutes. Remifentanil, as an opioid that acts on the selective $\mu$-receptor, has a more rapid onset time of within 1-2 minutes than other drug agents of the fentanyl group, and because it has a shorter half-life of within 5 minutes, since it is metabolized by a nonspecific esterase, it is quite useful for the inhibition 
of cardiovascular response to temporary stimulation such as endotracheal intubation [26]. Moreover, it can reduce the side effects of other opioids, such as long time low blood pressure and bradycardia, postoperative respiratory depression and delayed emergence [26]; thereby it can be useful for anesthesia of aged patients. Nonetheless, when co-administering with propofol, both drug agents display cardiovascular system inhibition actions, and they requires reduced clinical dose administration in order to prevent any severe low blood pressure. In particular, for the induction of anesthesia in aged patients, severe hemodynamic variation is expected due to the reduction of heart pulse output and the sensitivity decrease of the sympathetic nerve system's receptors; it is therefor crucial to pay attention to the cardiovascular system inhibition when using remifentanil. Kang and Lee [27] reported a gradual decrease of systolic and diastolic pressures in the group coadministered with remifentanil and propofol as well as in the group co-administered with remifentanil and midazolam, but the hemodynamic differences between the two groups were not significant, in connection with the effects of remifentanil on the cardiovascular actions of midazolam and propofol. Also, Ryu et al. [28] reported that when the central venous catheterization is used in pediatric patients, there was no significant difference in the intraoperative mean arterial pressure and in the intraoperative heart rate from both of the groups with the co-administration of remifentanil and midazolam, as well as the group with the co-administration of remifentanil and propofol. Therefore, in this study, the cardiovascular effects of remifentanil in the two groups can be assumed to be equal when it was administered under the same conditions, and it is considered that the administration of remifentanil in addition to propofol and midazolam had achieved unconsciousness, response blocking to harmful stimulation and amnesia by using the interactive synergistic actions between drug agents. Also those side effects including decrease of blood pressure, could be alleviated by reducing the respective administration doses of each drug.

As another method that can be to consider for reducing the decrease of blood pressure in aged patients for induction of anesthesia, propofol and remifentanil can be administered by target controlled infusion (TCI). From studies on this method, De Castro et al. [7] reported that remifentanil was a suitable drug agent for continuous intravenous administration due to its unique pharmacologic characteristic and that the TCI method in consideration of age, weight and heights in all patients was more effective for the maintenance of hemodynamic stability than the drug infusion based on body weight. Yim et al. [29] also reported that the continuous intravenous administration in the use of target effect-site concentration for the induction of anesthesia with the co-administration of remifentanil and propofol in aged patients has more hemodynamic stability than fast single intravenous bolus administration, since the former method had less incidences of severe low blood pressure before performing the endotracheal intubation. For another method, Chung and Lee [30] reported that if propofol is infused slowly at $20 \mathrm{mg} / \mathrm{kg} / \mathrm{hr}$ for the induction of anesthesia with a single use of propofol only in aged patients, the dose of $2.0 \mathrm{mg} / \mathrm{kg}$ does not cause a significant decrease of BP in comparison to the dose of $1.5 \mathrm{mg} / \mathrm{kg}$. In this study, even when the above drug agent was used by the single intravenous bolus administration, there was no case in which severe low blood pressure with systolic pressure below $70 \mathrm{mmHg}$ was presented. Therefore, it is preferable to use both of the two methods as aforementioned for the induction of general anesthesia if the operation theater has TCI equipment; but if TCI equipment is not available, it is considered that by performing the co-administration of propofol with TCI midazolam as suggested in this study, the induction of anesthesia in aged patients can be performed more safely.

In conclusion, when performing the induction of anesthesia in aged patients over 65 years old, using the combination of propofol and remifentanil, the co-induction with reduced dose of propofol to $0.8 \mathrm{mg} / \mathrm{kg}$ in combination with midazolam $0.3 \mathrm{mg} / \mathrm{kg}$ can prevent the incidence of prominent variation of blood pressure incurring before and after the endotracheal intubation.

\section{References}

1. Ryu KH. Critical point of anesthetic management in the elderly. Korean J Anesthesiol 2004; 46: 501-16.

2. Schnider TW, Minto CF, Shafer SL, Gambus PL, Andresen C, Goodale DB, et al. The influence of age on propofol pharmacodynamics. Anesthesiology 1999; 90: 1502-16.

3. Claeys MA, Gepts E, Camu F. Haemodynamic changes during anaesthesia induced and maintained with propofol. Br J Anaesth 1988; 60: 3-9.

4. Amrein R, Hetzel W, Allen SR. Co-induction of anaesthesia, the rationale. Eur J Anaesthesiol 1995; 12: 5-11.

5. Cressey DM, Claydon P, Bhaskaran NC, Reilly CS. Effect of midazolam pretreatment on induction dose requirements of propofol in combination with fentanyl in younger and older adults. Anaesthesia 2001; 56: 108-13.

6. Guignard B, Menigaux C, Dupont X, Fletcher D, Chauvin M. The effect of remifentanil on the bispectral index change and hemodynamic responses after orotracheal intubation. Anesth Analg 2000; 90: 161-7.

7. De Castro V, Godet G, Mencia G, Raux M, Coriat P. Target-controlled infusion for remifentanil in vascular patients improves hemodynamics and decreases remifentanil requirement. Anesth Analg 2003; 96: 33-8.

8. Kim CS, Shim JY, Ryu KH, Seo SW, Choi JH, Yang NY, et al. The effect of co-administration of midazolam on induction and recovery 
using continuous propofol infusion. Korean J Anesthesiol 1999; 37: 193-8.

9. Baraka AS, Taha SK, Aouad MT, El-Khatib MF, Kawkabani NI. Preoxygenation: comparison of maximal breathing and tidal volume breathing techniques. Anesthesiology 1999; 91: 612-6.

10. Ikeda T, Doi M, Morita K, Ikeda K. Effects of midazolam and diazepam as premedication on heart rate variability in surgical patients. Br J Anaesth 1994; 73: 479-83.

11. Paterniti S, Alperovitch A, Ducimetière P, Dealberto MJ, Lepine JP, Bisserbe JC. Anxiety but not depression is associated with elevated blood pressure in a community group of French elderly. Psychosom Med 1999; 61: 77-83.

12. Chang KS, Davis RF. Propofol produces endothelium independent vasodilatation and may act as a $\mathrm{Ca} 2+$ channel blocker. Anesth Analg 1993; 76: 24-32.

13. Kirkpatrick T, Cockshott ID, Douglas EJ, Nimmo WS. Pharmacokinetics of propofol (diprivan) in elderly patients. Br J Anaesth 1988; 60: $146-50$

14. Sebel PS, Lowdon JD. Propofol: a new intravenous anaesthetic. Anesthesiology 1989; 71: 260-77.

15. Charlson ME, MacKenzie CR, Gold JP, Ales KL, Topkins M, Fairclough GP Jr, et al. The preoperative and intraoperative hemodynamic predictors of postoperative myocardial infarction or ischemia in patients undergoing noncardiac surgery. Ann Surg 1989; 210: 637-48.

16. Schüttler J, Ihmsen H. Population phamacokinetics of propofol: a multicenter study. Anesthesiology 2000; 92: 727-38.

17. Olmos M, Ballester JA, Vidarte MA, Elizalde JL, Escobar A. The combined effect of age and premedication on the propofol requirements for induction by target-controlled infusion. Anesth Analg 2000; 90: 1157-61.

18. Nishiyama T, Matsukawa T, Hanaoka K. The effects of age and gender on the optimal premedication dose of intramuscular midazolam. Anesth Analg 1998; 86: 1103-8.

19. Lauven PM, Nadstawek J, Albrecht S. The safe use of anesthetics and muscle relaxants in older surgical patients. Drugs Aging 1993; 3: 502-9.
20. Platten HP, Schweizer E, Dilger K, Mikus G, Klotz U. Pharmacokinetics and the phamacodynamic action of midazolam in young and elderly patients undergoing tooth extraction. Clin Phamacol Ther 1998; 63: 552-60.

21. Greenblatt DJ, Abernethy DR, Locniskar A, Harmatz JS, Limjuco RA, Shader RI. Effect of age, gender, and obesity on midazolam kinetics. Anesthesiology 1984; 61: 27-35.

22. Kiefer RT, Weindler J, Ruprecht KW. Oral low-dose midazolam as premedication for intraocular surgery in retrobulbar anesthesia: cardiovascular effects and relief of perioperative anxiety. Eur J Ophthalmol 1997; 7: 185-92.

23. Cole SG, Brozinsky S, Isenberg JI. Midazolam, a new more potent benzodiazepine, compared with diazepam: a randomized doubleblind study of preendoscopic sedatives. Gastrointest Endosc 1983; 29: 219-22.

24. Short TG, Chui PT. Propofol and midazolam act synergistically in combination. Br J Anaesth 1991; 67: 539-45.

25. Teh J, Short TG, Wong J, Tan P. Pharmacokinetic interactions between midazolam and propofol: an infusion study. Br J Anaesth 1994; 72: 62-5.

26. Egan TD, Minto CF, Hermann DJ, Barr J, Muir KT, Shafer SL. Remifentanil versus alfentanil: comparative pharmacokinetics and pharmacodynamics in healthy adult male volunteers. Anesthesiology 1996; 84: 821-33.

27. Kang H, Lee HK. Changes of bispectral index and auditory evoked potential index during general anesthesia with propofol, midazolam and ketamine. Korean J Anesthesiol 2005; 49: 757-64

28. Ryu SH, Kwon JY, Lee HJ. The effects of midazolam or propofol combined with remifentanil infusion for central venous catheterization in children. Korean J Anesthesiol 2007; 52: 669-74

29. Yim EB, Lee GY, Han JI, Chung RK. Hemodynamic changes between different remifentanil administration methods during induction in the elderly. Korean J Anesthesiol 2007; 53: 714-9.

30. Chung AL, Lee GY. BIS and hemodynamic change in elderly patients by propofol infusion for induction. Korean J Anesthesiol 2006; 50: 417-21. 